\title{
Contaminación por mohos y aislamiento de Cladosporium spp. y Thamnidium spp. en cámaras frigoríficas de plantas procesadoras de alimentos marinos y empresas frigoríficas del sur de Chile
}

\author{
(Mould contamination and isolation of Cladosporium spp. and Thamnidium \\ spp. in frigorific chambers of sea-food processing plants \\ and frigorific enterprises from South of Chile)
}

Boris Castillo B ${ }^{1 *}$, Carlos Díaz A $^{1}$,Pablo Santibañez ${ }^{1}$ G.

1. Inocuidad Alimentaria, Aquagestión S.A. 5550000.

*Autor para correspondencia: boris.castillo.bascur@gmail.com

RECIBIDO: 02 de Febrero de 2017

APROBADO: 18 de Mayo de 2017

DOI: $10.22370 /$ bolmicol.2017.32.1.628

LOS AUTORES DECLARAN NO TENER CONFLICTO DE INTERESES

Palabras claves: Cladosporium; Thamnidium; frigorífico; moho.

Key words: Cladosporium; Thamnidium; frigorific; mould.

\section{RESUMEN}

La apertura de nuevos mercados para los productos hidrobiológicos chilenos ha impuesto el análisis del estatus sanitario de cámaras frigoríficas donde estos productos son almacenados. Para medir el estatus sanitario, la Unión Económica Euroasiática (UEE) exige un recuento total de mohos e identificación de los géneros Cladosporium y Thamnidium en paredes y ambiente de las cámaras frigoríficas. Poco se sabe sobre los niveles de contaminación de mohos en cámaras frigoríficas de productos de exportación en Chile. Aquí, los resultados de 88 análisis de mohos en paredes y aire, realizados a 24 empresas diferentes que poseen cámaras frigoríficas conteniendo productos hidrobiológicos, son mostrados. El recuento total de mohos y el número de colonias de Cladosporium spp. y Thamnidium spp. fueron determinados. Además, los niveles de contaminación por mohos fueron comparados entre plantas procesadoras de alimentos y empresas frigoríficas. Thamnidium spp. no fue encontrado en ninguno de los análisis realizados. Los resultados indican que los niveles de contaminación por mohos son aceptables para la UEE. Este es el primer reporte de niveles de contaminación de mohos en cámaras frigoríficas de productos hidrobiológicos en el sur de Chile.

\section{ABSTRACT}

The opening of new markets for Chilean hydrobiological products had imposed the analysis of the sanitary status of frigorific chambers where those products are kept. To measure the sanitary status, the Euroasiatic Economical Union (EEU) requires a total mould count and identification of 
Cladosporium and Thamnidium in walls and air of the frigorific chambers. Little is known about contamination levels of moulds in frigorific chambers of export products in Chile. Here, the results of 88 mould analyses in walls and air, performed to 24 different enterprises that possess frigorific chambers containing hydrobiological products, are shown. Total mould count and Cladosporium spp. and Thamnidium spp. colony number were determined. Additionally the levels of moulds contamination were compared between food-processing plants and frigorific enterprises. Thamnidium spp. was not found in any of the analyses performed. The results indicate that levels of moulds contamination are acceptable to the EEU. This is the first report of mould contamination levels in frigorific chambers of hydrobiological products from south of Chile.

\section{INTRODUCCIÓN}

Chile es uno de los principales exportadores de salmones y otros productos hidrobioló$\operatorname{gicos}^{1}$. La cadena productiva abastece a diversos mercados de los 5 continentes. La necesidad de solventar la exportación de productos hidrobiológicos y el esfuerzo de permanecer como líder mundial en esta materia, ha impuesto la habilitación de infraestructura sofisticada que garantice una correcta explotación y comercialización de los productos. En este sentido, las cámaras frigoríficas son espacios que adquieren un rol fundamental para asegurar la calidad e inocuidad de los alimentos desde que son cosechados hasta que son enviados a los mercados de destino. Todas las plantas de proceso de productos hidrobiológicos poseen, al menos, una cámara frigorífica que garantiza la cadena de frío y preservación. La cantidad de productos a comercializar es tan alta que se han ido creando, desde varios años atrás, empresas dedicadas sólo a mantener productos refrigerados, con sofisticados sistemas de control, gestión y regulación; los llamados frigoríficos.
En la actualidad, la Unión Económica Euroasiática (UEE) exige a sus proveedores de productos pesqueros realizar un análisis de mohos en las cámaras frigoríficas que participan en la cadena de producción, mantención y transporte de los productos pesqueros hasta que arriban a la $\mathrm{UEE}^{2}$. Este ensayo consiste en un recuento del total de mohos en paredes y ambiente de las cámaras, junto con la identificación y recuento de los géneros Cladosporium y Thamnidium. El motivo de realizar este análisis es evaluar el estado sanitario de las cámaras frigoríficas, pudiendo ser bueno, satisfactorio o malo. Las empresas deben poseer protocolos internos de medidas correctivas en caso de calificación mala, o intensificar las rutinas de aseo cuando la calificación es satisfactoria.

Cladosporium y Thamnidium son dos géneros de hongos conocidos por participar en el proceso de descomposición de alimentos, siendo particularmente relevantes en la descomposición a bajas temperaturas $\left(0^{\circ} \mathrm{C}\right)^{3}$. Ambos mohos son encontrados comúnmente en aire de muchos ambientes. En general, los mohos son utilizados como un buen marcador de estado sanitario y evaluación de protocolos de aseo en ambientes cerra$\operatorname{dos}^{4}$.

Poco se conoce de la presencia de mohos en cámaras frigoríficas en Chile, aún menos en aquellas destinadas a almacenar productos pesqueros de exportación. El presente trabajo expone los resultados de una serie de análisis de contaminación por mohos realizados a cámaras frigoríficas de plantas de proceso de productos pesqueros $\mathrm{y}$ empresas frigoríficas durante junio y diciembre de 2016 en el sur de Chile (X a XII Región), regiones donde ocurre el mayor volumen de explotación de productos hidrobiológicos y es el primer reporte de niveles de contaminación por mohos e identificación de Cladosporium spp. y Thamnidium spp. en cámaras frigoríficas de productos hidrobiológicos en el sur de Chile. 


\section{MATERIALES Y MÉTODOS}

Tamaño de la muestra y macrozonas. Un total de 88 análisis de detección de mohos en paredes y ambiente fueron realizados a 24 empresas diferentes de la zona sur de Chile. Las empresas fueron divididas en 3 diferentes macrozonas (Figura 1) de acuerdo al sitio respectivo de ubicación de sus cámaras frigoríficas. Las empresas fueron clasificadas como "Plantas de proceso" y "Frigorífico" de acuerdo a su actividad principal (Tabla 1).

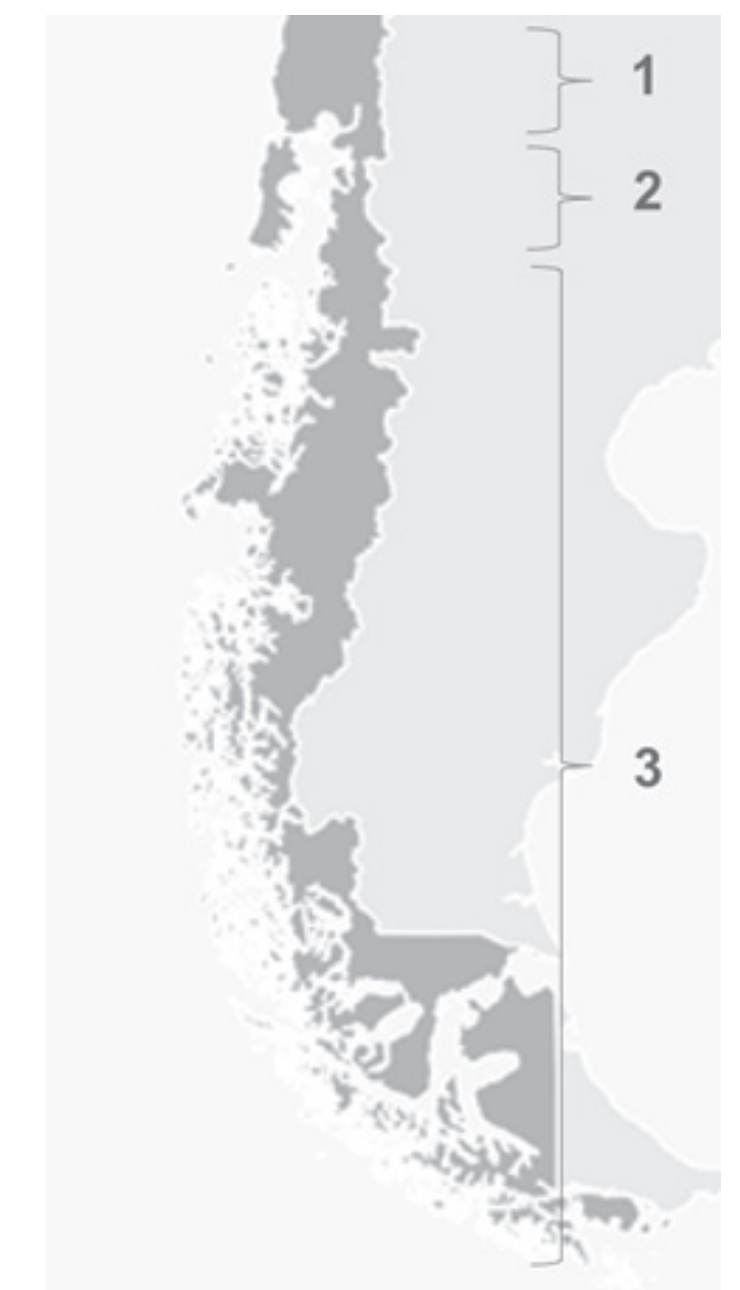

Figura 1. Distribución geográfica de macrozonas del sur de Chile analizadas. La macrozona 1 comprende la localización sur de las provincias de Llanquihue. Macrozona 2 comprende la provincia de Chiloé, y la macrozona 3 las regiones XI y XII.
Toma de muestras de paredes de las cámaras frigoríficas. La toma de muestras de paredes de las cámaras frigoríficas fue realizada utilizando esponjas hidratadas $\left(3 \mathrm{M}^{\circledR}\right)$, abarcando una superficie de $5 \mathrm{~cm}^{2}$ a 1,5 metros de altura del piso, en las 4 paredes de la cámara. Las 4 esponjas obtenidas por cada cámara (4 paredes) fueron mezcladas con 60 $\mathrm{ml}$ de agua destilada estéril, y se homogenizaron por 3 minutos para liberar los microorganismos adheridos. Finalmente, $1 \mathrm{ml}$ de la mezcla anterior fue sembrado en profundidad en agar Sabouraud (Merck ${ }^{\circledR}$ ) e incubado a $24^{\circ} \mathrm{C}$ por 7 días para realizar recuento de mohos e identificación de miembros del género Cladosporium y Thamnidium. La incubación se realizó a $24^{\circ} \mathrm{C}$, porque es la temperatura de incubación indicada en el protocolo técnico de la normativa rusa aplicable a este tipo de análisis ${ }^{2}$.

Toma de muestras de ambiente de las cámaras frigoríficas. La toma de muestra de ambiente de las cámaras fue realizada exponiendo por 5 minutos placas con agar Sabouraud, una placa en cada una de las cuatro esquinas de la cámara y una placa en el centro. Transcurrido el tiempo de exposición, las placas fueron transportadas selladas a $2-4^{\circ} \mathrm{C}$ e incubadas a $24^{\circ} \mathrm{C}$ por 7 días. La incubación se realizó a $24^{\circ} \mathrm{C}$, porque es la temperatura de incubación indicada en el protocolo técnico de la normativa rusa aplicable a este tipo de análisis².

Recuento total de mohos. El recuento total de mohos fue realizado luego de 7 días de incubación, tanto para las muestras de paredes como de ambiente. Los mohos fueron contabilizados por recuento directo en placa de acuerdo a las características típicas de crecimiento y morfología de los mohos. Se realizó recuento de colonias de Penicillium spp. al día 3, las que fueron marcadas, con el fin de evitar recuento de colonias secundarias al día 7 .

\section{Identificación de Cladosporium y Thamnidium.} La identificación de Cladosporium spp. y Thamnidium spp. fue realizada por morfología macro y microscópica, utilizando claves taxonómicas ${ }^{3}$, hasta 
el nivel de género. Con cinta adhesiva, la colonia sospechosa del moho fue impresa suavemente. Posteriormente, dicha cinta fue pegada sobre un portaobjeto conteniendo una gota de azul de lactofenol $\left(\right.$ Merck $\left.^{\circledR}\right)$. El género fue confirmado por exploración a $400 \mathrm{X}$ en microscopio óptico BM -700 $\left(\right.$ Boeco $\left.^{\circledR}\right)$.
Análisis y procesamiento de datos. En las tablas de resultados, lo indicado como "cantidad afectados", indica el número de análisis que poseen recuentos de mohos mayores a 0 . Los datos fueron procesados y analizados estadísticamente mediante ANOVA y test de Tukey, utilizando el software SigmaPlot 11 (Systat Software $^{\circledR}$ ).

Tabla 1. Distribución de macrozonas, ciudades, cantidad y tipo de empresas analizadas.

\begin{tabular}{|c|c|c|c|c|}
\hline Macrozona & Ciudad & Total & Planta de Proceso & Frigorífico \\
\hline \multirow{3}{*}{1} & Puerto Varas & 1 & 0 & 1 \\
\cline { 2 - 5 } & Puerto Montt & 12 & 8 & 4 \\
\cline { 2 - 5 } & Calbuco & 1 & 1 & 0 \\
\hline \multirow{3}{*}{2} & Castro & 1 & 1 & 0 \\
\cline { 2 - 5 } & Chonchi & 1 & 1 & 0 \\
\cline { 2 - 5 } & Quellón & 4 & 4 & 0 \\
\hline \multirow{3}{*}{3} & Puerto Chacabuco & 2 & 2 & 5 \\
\cline { 2 - 5 } & Punta Arenas & 2 & 2 & 0 \\
\hline
\end{tabular}

\section{RESULTADOS Y DISCUSIÓN}

Contaminación por mohos de acuerdo a macrozona. Se observa que los niveles de mohos en las 3 macrozonas es similar, tanto en paredes como en ambiente (Tabla 2). No hay diferencias significativas entre los recuentos de mohos en paredes ni ambiente en las distintas macrozonas (test de Tukey). El promedio de colonias de mohos no es estadísticamente diferente entre las distintas macrozonas, y paredes y ambiente de las cámaras frigoríficas.

La Figura 2 muestra el número de colonias y la cantidad de análisis realizados que presentaron dicho número de colonias, desglosado en paredes y ambiente. Se observa que la mayor parte de los análisis exhiben recuentos de 0 a 2 ufc. Sin embargo, hay casos puntuales donde se obtuvieron recuentos $>10$ ufc. Esto indica que existen diversos factores que afectan el nivel de contaminación por mohos de las cámaras frigoríficas, que impiden niveles de contaminación homogénea, y además influencian el promedio y desviación estándar de los datos. Estos factores pueden ser: tecnología de las cámaras, posición geográfica, frecuencia de ingreso y egreso de productos, estados de pallets de carga, etc.

Presencia de Cladosporium y Thamnidium de acuerdo a macrozona. Cladosporium se encuentra preferentemente contaminando el ambiente de las cámaras frigoríficas, con una cantidad de análisis afectados superior al $15 \%$ de las cámaras 
Tabla 2. Casos positivos y recuento promedio de colonias de mohos totales en las distintas macrozonas.

\begin{tabular}{|c|c|c|c|c|c|c|c|}
\hline \multirow[b]{2}{*}{ Macrozona } & \multirow[b]{2}{*}{$\mathrm{N}^{\circ}$ análisis } & \multicolumn{3}{|c|}{ Paredes } & \multicolumn{3}{|c|}{ Ambiente } \\
\hline & & $\begin{array}{l}\text { Cantidad } \\
\text { afectados }\end{array}$ & $\begin{array}{c}\text { Cantidad } \\
\text { afectados }(\%)\end{array}$ & $\begin{array}{c}\text { Promedio } \\
\text { colonias } \\
\left(\mathrm{ufc} / \mathrm{cm}^{2}\right)\end{array}$ & $\begin{array}{l}\text { Cantidad } \\
\text { afectados }\end{array}$ & $\begin{array}{c}\text { Cantidad } \\
\text { afectados } \\
(\%)^{*}\end{array}$ & $\begin{array}{c}\text { Promedio } \\
\text { colonias } \\
\text { (ufc/placa/5 } \\
\text { min) }\end{array}$ \\
\hline 1 & 64 & 36 & 56,3 & $3,9( \pm 3,3)$ & 41 & 64,1 & $4,6( \pm 4,3)$ \\
\hline 2 & 13 & 4 & 30,8 & $2,5( \pm 1,7)$ & 9 & 69,2 & $2,3( \pm 1,7)$ \\
\hline 3 & 11 & 3 & 27,3 & $8,0( \pm 12,1)$ & 8 & 72,7 & $4,0( \pm 4,1)$ \\
\hline
\end{tabular}

* La cantidad de afectados refleja el porcentaje de análisis que poseen un recuento total de mohos $>1$ ufc por unidad de medida con respecto a la cantidad total de análisis realizados por macrozona.

analizadas (Tabla 3). El promedio de colonias de Cladosporium es de 1 ufc tanto en paredes como ambiente. En las macrozonas 2 y 3 no se detectó presencia de Cladosporium en paredes (Tabla 3). Esto se podría deber que la cantidad de análisis realizado a distintas empresas en cada macrozona no es igual, por lo que los recuentos de Cladosporium pueden estar subvaloradas. Thamnidium, en cambio, no se encontró en ninguno de los análisis efectuados, ni en ambiente ni en paredes de ninguna macrozona (Tabla 4).
Comparación de recuentos de mohos totales por tipo de empresa. Hay diferencias significativas entre ambiente de frigorífico y planta de proceso (p-value: 0,0010$)$ en relación a recuento de mohos totales. En cambio, en el caso de las paredes, no se observa diferencias significativas entre las plantas de proceso y los frigoríficos ( $\mathrm{p}$-value: 0,3828) (Tabla 5).

Estos resultados concuerdan con el hecho que las empresas frigoríficas poseen cámaras de

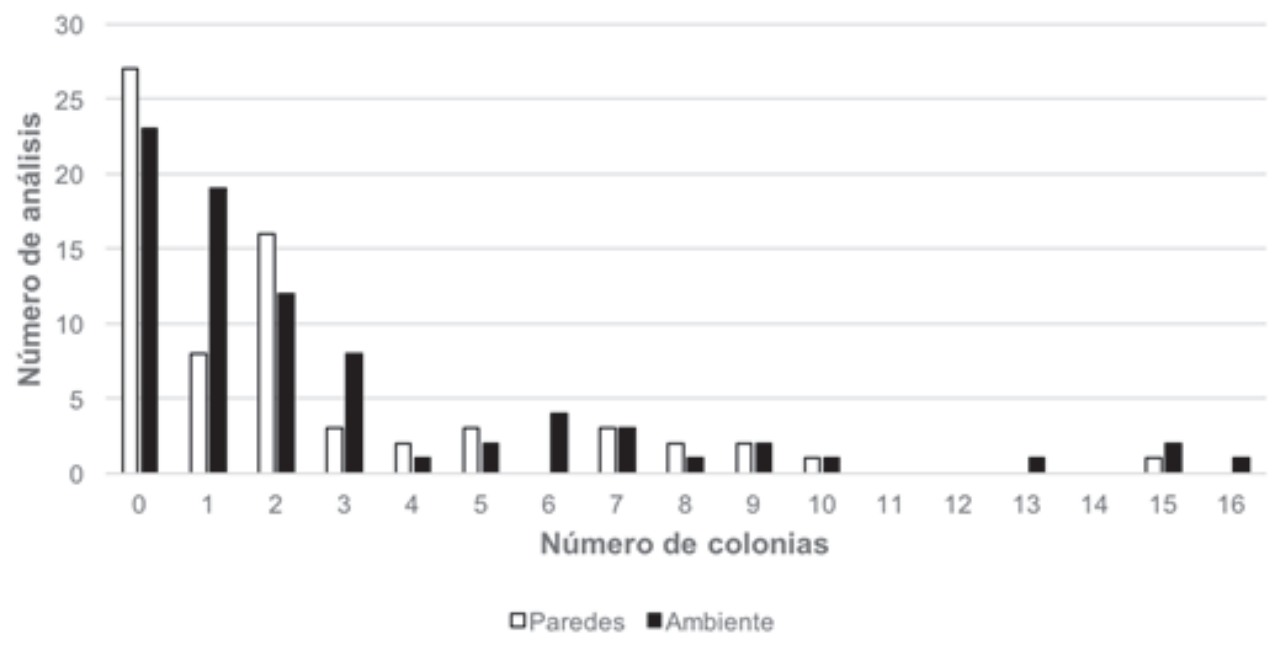

Figura 2. Distribución del número de colonias de acuerdo a la cantidad de análisis realizados con dicho recuento. Barras blancas y negras son el número de colonias en paredes y ambiente, respectivamente. 
una dimensión mucho mayor a las cámaras frigoríficas de las Plantas de Proceso, por lo que los volúmenes de aire que circulan dentro son más altos. También, la diversidad y cantidad de productos almacenados en Frigoríficos es mayor, por lo que la contribución a mohos ambientales proveniente de cajas y pallets de madera, debería ser mayor también. De todas formas, estas hipótesis deben ser demostradas en estudios posteriores.

Comparación de recuentos de Cladosporium por tipo de empresa. La Tabla 6 muestra una pre- sencia más elevada de Cladosporium en ambiente que en paredes. Esto se observa tanto en plantas de proceso como frigoríficos. Este resultado es esperable, ya que Cladosporium posee conidias adaptadas a permanecer en aire ${ }^{5}$. Los niveles de contaminación por Cladosporium no presentaron diferencias significativas entre los dos tipos de empresa ni entre paredes y ambiente. Ningún representante del género Thamnidium fue encontrado en los análisis realizados, por lo que no se muestran los resultados. Los resultados son congruentes con los observado en la Tabla 3 y 4.

Tabla 3. Promedio de colonias de Cladosporium por macrozona.

\begin{tabular}{|c|c|c|c|c|c|c|c|}
\hline \multirow[b]{2}{*}{ Macrozona } & \multirow[b]{2}{*}{$\mathrm{N}^{\circ}$ análisis } & \multicolumn{3}{|c|}{ Paredes } & \multicolumn{3}{|c|}{ Ambiente } \\
\hline & & $\begin{array}{l}\text { Cantidad } \\
\text { afectados }\end{array}$ & $\begin{array}{c}\text { Cantidad } \\
\text { afectados }(\%) \text { * }\end{array}$ & $\begin{array}{l}\text { Promedio } \\
\text { colonias } \\
\left(u f c / \mathrm{cm}^{2}\right)\end{array}$ & $\begin{array}{l}\text { Cantidad } \\
\text { afectados }\end{array}$ & $\begin{array}{c}\text { Cantidad } \\
\text { afectados } \\
(\%)^{*}\end{array}$ & $\begin{array}{c}\text { Promedio } \\
\text { colonias } \\
\text { (ufc/placa/5 } \\
\text { min) }\end{array}$ \\
\hline 1 & 64 & 4 & 6,4 & $1( \pm 1)$ & 16 & 25,4 & $2( \pm 1)$ \\
\hline 2 & 13 & 0 & 0 & 0 & 2 & 15,4 & $1( \pm 1)$ \\
\hline 3 & 11 & 0 & 0 & 0 & 2 & 18,2 & $1( \pm 1)$ \\
\hline
\end{tabular}

* La cantidad de afectados refleja el porcentaje de análisis que poseen $>1$ ufc de Cladosporium spp. por unidad de medida con respecto a la cantidad total de análisis realizados por macrozona.

Tabla 4. Promedio de colonias de Thamnidium por macrozona.

\begin{tabular}{|c|c|c|c|c|c|c|c|}
\hline \multirow[b]{2}{*}{ Macrozona } & \multirow[b]{2}{*}{$\mathrm{N}^{\circ}$ análisis } & \multicolumn{3}{|c|}{ Paredes } & \multicolumn{3}{|c|}{ Ambiente } \\
\hline & & $\begin{array}{l}\text { Cantidad } \\
\text { afectados }\end{array}$ & $\begin{array}{c}\text { Cantidad } \\
\text { afectados }(\%) \text { * }\end{array}$ & $\begin{array}{c}\text { Promedio } \\
\text { colonias } \\
\left(\mathrm{ufc} / \mathrm{cm}^{2}\right)\end{array}$ & $\begin{array}{l}\text { Cantidad } \\
\text { afectados }\end{array}$ & $\begin{array}{c}\text { Cantidad } \\
\text { afectados } \\
(\%)^{*}\end{array}$ & $\begin{array}{l}\text { Promedio } \\
\text { colonias } \\
\text { (ufc/placa/5 } \\
\text { min) }\end{array}$ \\
\hline 1 & 64 & 0 & 0 & 0 & 0 & 0 & 0 \\
\hline 2 & 13 & 0 & 0 & 0 & 0 & 0 & 0 \\
\hline 3 & 11 & 0 & 0 & 0 & 0 & 0 & 0 \\
\hline
\end{tabular}

* La cantidad de afectados refleja el porcentaje de análisis que poseen $>1$ ufc de Thamnidium spp. por unidad de medida con respecto a la cantidad total de análisis realizados por macrozona. 
Tabla 5. Comparación de recuentos promedios de mohos totales entre Plantas de Proceso y Frigoríficos.

\begin{tabular}{|c|c|c|c|c|c|c|c|}
\hline \multirow[b]{2}{*}{$\begin{array}{l}\text { Tipo de } \\
\text { Empresa }\end{array}$} & \multirow[b]{2}{*}{$\begin{array}{c}\mathrm{N}^{\circ} \\
\text { análisis }\end{array}$} & \multicolumn{3}{|c|}{ Paredes } & \multicolumn{3}{|c|}{ Ambiente } \\
\hline & & $\begin{array}{l}\text { Cantidad } \\
\text { afectados }\end{array}$ & $\begin{array}{c}\text { Cantidad } \\
\text { de } \\
\text { afectados } \\
(\%)^{*}\end{array}$ & $\begin{array}{c}\text { Promedio } \\
\text { colonias } \\
\left(\mathrm{ufc} / \mathrm{cm}^{2}\right)\end{array}$ & $\begin{array}{l}\text { Cantidad } \\
\text { afectados }\end{array}$ & $\begin{array}{c}\text { Cantidad } \\
\text { de } \\
\text { afectados } \\
(\%)^{*}\end{array}$ & $\begin{array}{c}\text { Promedio } \\
\text { colonias } \\
\text { (ufc/placa/5 } \\
\text { min) }\end{array}$ \\
\hline $\begin{array}{c}\text { Planta de } \\
\text { Proceso }\end{array}$ & 40 & 20 & 50,0 & $3,5( \pm 2,7)$ & 22 & 55,0 & $2,6( \pm 2,2)$ \\
\hline Frigorífico & 24 & 16 & 66,7 & $4,4( \pm 4,0)$ & 19 & 79,2 & $6,8( \pm 5,0)$ \\
\hline
\end{tabular}

* La cantidad de afectados refleja el porcentaje de análisis que poseen un recuento total de mohos $>1$ ufc por unidad de medida con respecto al tipo de empresa.

Tabla 6. Comparación de recuentos promedios de colonias de Cladosporium entre Plantas de Proceso y Frigoríficos de la macrozona 1.

\begin{tabular}{|c|c|c|c|c|c|c|c|}
\hline \multirow[b]{2}{*}{$\begin{array}{l}\text { Tipo de } \\
\text { Empresa }\end{array}$} & \multirow[b]{2}{*}{$\begin{array}{c}\mathrm{N}^{0} \\
\text { análisis }\end{array}$} & \multicolumn{3}{|c|}{ Paredes } & \multicolumn{3}{|c|}{ Ambiente } \\
\hline & & $\begin{array}{l}\text { Cantidad } \\
\text { afectados }\end{array}$ & $\begin{array}{c}\text { Cantidad } \\
\text { de } \\
\text { afectados } \\
(\%)^{*}\end{array}$ & $\begin{array}{c}\text { Promedio } \\
\text { colonias } \\
\left(\mathrm{ufc} / \mathrm{cm}^{2}\right)\end{array}$ & $\begin{array}{l}\text { Cantidad } \\
\text { afectados }\end{array}$ & $\begin{array}{l}\text { Cantidad } \\
\text { de } \\
\text { afectados } \\
(\%)^{*}\end{array}$ & $\begin{array}{c}\text { Promedio } \\
\text { colonias } \\
\text { (ufc/placa/5 } \\
\text { min) }\end{array}$ \\
\hline $\begin{array}{l}\text { Planta de } \\
\text { Proceso }\end{array}$ & 40 & 20 & 50,0 & $3,5( \pm 2,7)$ & 22 & 55,0 & $2,6( \pm 2,2)$ \\
\hline Frigorífico & 24 & 16 & 66,7 & $4,4( \pm 4,0)$ & 19 & 79,2 & $6,8( \pm 5,0)$ \\
\hline
\end{tabular}

* La cantidad de afectados refleja el porcentaje de análisis que poseen $>1$ ufc de Cladosporium spp. por unidad de medida con respecto al tipo de empresa.

\section{CONCLUSIONES}

La contaminación por mohos en cámaras frigoríficas de productos hidrobiológicos del sur de Chile es baja, estando, en la mayoría de los casos, dentro del rango bueno y satisfactorio de la normativa rusa sanitaria de frigoríficos ${ }^{2}$. Por su parte, aunque el porcentaje de análisis con presencia de Cladosporium spp. es alto, el recuento promedio de mohos de este género en cada uno de los análisis es bajo. Esto significa que las cámaras en promedio, aun teniendo recuentos de Cladosporium, deberían estar dentro o por sobre el rango de clasificación satisfactorio de la normativa rusa. Thamnidium, en cambio, no fue encontrado en ninguno de los análisis realizados.

Los resultados aqui presentados son el primer reporte de recuentos promedios de mohos e identificación de Cladosporium spp. y Thamnidium spp. en cámaras frigoríficas de productos hidrobiológicos del sur de Chile. Se muestra una tendencia general de la realidad en las cámaras frigoríficas en relación a los mohos, pudiendo existir otros antecedentes importantes que no fueron considerados para este estudio y que podrían afectar los resulta- 
dos, como el tipo de terreno de emplazamiento de las cámaras (costas, praderas, presencia de bosques o plantaciones cercanas, etc.); correlación de época del año y variables ambientales (vientos, lluvias, temperatura); o la tecnología de las cámaras (presencia de filtros de aire, estado de evaporizadores, etc.).
Finalmente, los resultados aquí presentados pueden servir como base de referencia para las empresas que necesitan analizar mohos en sus cámaras frigoríficas, o para definir políticas públicas con respecto a este tema por parte de los organismos pertinentes del Estado.

\section{REFERENCIAS}

1. FAO. El estado mundial de la pesca y la acuicultura 2016. Contribución a la seguridad alimentaria y la nutrición para todos. Roma: FAO; 2016. [citado 2 Ene 2017]. Disponible en: http://www.fao. org/3/a-i5555s.pdf.

\section{Servicio Nacional de Pesca y Acuicultura.} Normas para Frigoríficos (ES). Chile: Sernapesca; 2015 [actualizado 15 Oct 2015; citado 2 Ene 2017]. Disponible en: http://www.sernapesca.cl/ index.php?option $=$ com_remository\&Itemid $=246$ $\&$ func $=$ fileinfo\&id $=14561$.

3. Pitt JI, Hocking AD. Fungi and Food Spoilage. 3th ed. New York: Springer. 2009 [citado 2 Ene
2017]. Disponible en: http://link.springer.com/boo k/10.1007\%2F978-0-387-92207-2

4. WHO. WHO guidelines for indoor air quality: dampness and moulds. Dinamarca: WHO; 2009. [citado 5 Ene 2017]. Disponible en: http://www. euro.who.int/_data/assets/pdf_file/0017/43325/ E92645.pdf.

5. Lacey $\mathbf{J}$. The aerobiology of conidial fungi. In: Core GT, Kendrick B, editors. Biology of Conidial Fungi. New York: Academic Press; 1981. p. 373-416. [citado 5 Ene 2017]. Disponible en: http://www.sciencedirect.com/science/ book/9780121795016 